\title{
Topological loops with six-dimensional solvable multiplication groups having five-dimensional nilradical*
}

\author{
Ágota Figula, Kornélia Ficzere, Ameer Al-Abayechi \\ University of Debrecen, Institute of Mathematics, Hungary \\ figula@science.unideb.hu \\ ficzerelia@gmail.com \\ ameer@science.unideb.hu \\ Submitted: July 15, 2019 \\ Accepted: August 4, 2019 \\ Published online: August 14, 2019
}

\begin{abstract}
Using connected transversals we determine the six-dimensional indecomposable solvable Lie groups with five-dimensional nilradical and their subgroups which are the multiplication groups and the inner mapping groups of three-dimensional connected simply connected topological loops. Together with this result we obtain that every six-dimensional indecomposable solvable Lie group which is the multiplication group of a three-dimensional topological loop has one-dimensional centre and two- or three-dimensional commutator subgroup.
\end{abstract}

Keywords: multiplication group of a topological loop, connected transversals, linear representations of solvable Lie algebras

$M S C:$ 22E25, 17B30, 20N05, 57S20, 53C30

\section{Introduction}

The multiplication group $\operatorname{Mult}(L)$ and the inner mapping group $\operatorname{Inn}(L)$ of a loop $L$ are important tools for the investigations in loop theory since there are strong

*The paper was supported by the EFOP-3.6.1-16-2016-00022 project. This projects have been supported by the European Union, co-financed by the European Social Fund. 
relations between the structure of the normal subloops of $L$ and that of the normal subgroups of $\operatorname{Mult}(L)$ (cf. [1, 2]). In [9] the authors have obtained necessary and sufficient conditions for a group $G$ to be the multiplication group of $L$. These conditions say that one can use special transversals $A$ and $B$ with respect to a subgroup $K$ of $G$. The subgroup $K$ plays the role of the inner mapping group of $L$ whereas the transversals $A$ and $B$ belong to the sets of left and right translations of $L$.

P. T. Nagy and K. Strambach in [8] investigate thoroughly topological and differentiable loops as continuous and differentiable sections in Lie groups. In this paper we follow their approach and study topological loops $L$ of dimension 3 having a solvable Lie group as their multiplication group. Applying the criteria of [9] we obtained in [3] all solvable Lie groups of dimension $\leq 5$ which are the multiplication group of a 3-dimensional connected simply connected topological proper loop. This classification has resulted only decomposable Lie groups as the group $\operatorname{Mult}(L)$ of $L$. Hence we paid our attention to 6 -dimensional solvable indecomposable Lie groups. If their Lie algebras have a 4-dimensional nilradical, then among the 40 isomorphism classes of Lie algebras there is only one class depending on a real parameter which consists of the Lie algebras of the group $\operatorname{Mult}(L)$ of $L$ (cf. [4]). This result has confirmed the observation that the condition for the multiplication group of a topological loop to be a (finite-dimensional) Lie group is strong. Since the 6-dimensional solvable indecomposable Lie algebras have 4 or 5 -dimensional nilradical it remains to deal with the 99 classes of solvable Lie algebras having 5dimensional nilradical (cf. [7, 10]). In [5] we proved that among them there are 20 classes of Lie algebras which satisfy the necessary conditions to be the Lie algebra of the group $\operatorname{Mult}(L)$ of a 3-dimensional loop $L$. We determined there also the possible subalgebras of the corresponding inner mapping groups.

The purpose of this paper is to determine the indecomposable solvable Lie groups of dimension 6 which have 5-dimensional nilradical and which are the multiplication group of a 3-dimensional connected simply connected topological loop. To find a suitable linear representation of the simply connected Lie groups for the 20 classes of solvable Lie algebras given in [5] is the first step to achieve this classification (cf. Theorem 3.1). Applying the method of connected transversals we show that only those Lie groups $G$ in Theorem 3.1 which have 2- or 3-dimensional commutator subgroup allow continuous left transversals $A$ and $B$ in the group $G$ with respect to the subgroup $K$ given in Theorem 3.1 such that $A$ and $B$ are $K$-connected and $A \cup B$ generates $G$ (cf. Proposition 3.2 and Theorem 3.3). An arbitrary left transversal $A$ to the 3 -dimensional abelian subgroup $K$ of $G$ depends on three continuous real functions with three variables. The condition that the left transversals $A$ and $B$ are $K$-connected is formulated by functional equations. Summarizing the results of Theorem in [6], of Theorem 16 in [4] and of Theorem 3.3 we obtain that each 6 -dimensional solvable indecomposable Lie group which is the multiplication group of a 3-dimensional topological loop has 1-dimensional centre and two- or three-dimensional commutator subgroup. 


\section{Preliminaries}

A loop is a binary system $(L, \cdot)$ if there exists an element $e \in L$ such that $x=$ $e \cdot x=x \cdot e$ holds for all $x \in L$ and the equations $x \cdot a=b$ and $a \cdot y=b$ have precisely one solution $x=b / a$ and $y=a \backslash b$. A loop is proper if it is not a group.

The left and right translations $\lambda_{a}=y \mapsto a \cdot y: L \rightarrow L$ and $\rho_{a}: y \mapsto y \cdot a: L \rightarrow L$, $a \in L$, are bijections of $L$. The permutation group $\operatorname{Mult}(L)=\left\langle\lambda_{a}, \rho_{a} ; a \in L\right\rangle$ is called the multiplication group of $L$. The stabilizer of the identity element $e \in L$ in $\operatorname{Mult}(L)$ is called the inner mapping group $\operatorname{Inn}(L)$ of $L$.

Let $G$ be a group, let $K \leq G$, and let $A$ and $B$ be two left transversals to $K$ in $G$. We say that $A$ and $B$ are $K$-connected if $a^{-1} b^{-1} a b \in K$ for every $a \in A$ and $b \in B$. The core $C o_{G}(K)$ of $K$ in $G$ is the largest normal subgroup of $G$ contained in $K$. If $L$ is a loop, then $\Lambda(L)=\left\{\lambda_{a} ; a \in L\right\}$ and $R(L)=\left\{\rho_{a} ; a \in L\right\}$ are $\operatorname{Inn}(L)$-connected transversals in the group $\operatorname{Mult}(L)$ and the core of $\operatorname{Inn}(L)$ in $\operatorname{Mult}(L)$ is trivial. In [9], Theorem 4.1, the following necessary and sufficient conditions are established for a group $G$ to be the multiplication group of a loop $L$ :

Proposition 2.1. A group $G$ is isomorphic to the multiplication group of a loop if and only if there exists a subgroup $K$ with $C_{G}(K)=1$ and $K$-connected transversals $A$ and $B$ satisfying $G=\langle A, B\rangle$.

A loop $L$ is called topological if $L$ is a topological space and the binary operations $(x, y) \mapsto x \cdot y,(x, y) \mapsto x \backslash y,(x, y) \mapsto y / x: L \times L \rightarrow L$ are continuous. In general the multiplication group of a topological loop $L$ is a topological transformation group that does not have a natural (finite dimensional) differentiable structure. In this paper we deal with 3 -dimensional connected simply connected topological loops $L$. We assume that the multiplication group of $L$ is a 6 -dimensional solvable indecomposable Lie group $G$ such that its Lie algebra has 5-dimensional nilradical. Then $L$ is homeomorphic to $\mathbb{R}^{3}$ (cf. [3, Lemma 5]). Since it has nilpotency class 2 (cf. [5, Theorem 3.1]) by Theorem $8 \mathrm{~A}$ in [2] the subgroup $K$ in Proposition 2.1 is a 3-dimensional abelian Lie subgroup of $G$ which does not contain any non-trivial normal subgroup of $G, A$ and $B$ are continuous $K$-connected left transversals to $K$ in $G$ such that $A \cup B$ generates $G$.

\section{Six-dimensional solvable Lie multiplication groups with five-dimensional nilradical}

Using necessary conditions we found in [5], Theorems 3.6, 3.7, those 6-dimensional solvable indecomposable Lie algebras with 5-dimensional nilradical which can occur as the Lie algebra $\mathbf{g}$ of the multiplication group of a 3-dimensional topological loop $L$. We obtained also the Lie subalgebras $\mathbf{k}$ of the inner mapping group of $L$. With the notation in [10] they are the following:

$\mathbf{g}_{1}:=\mathbf{g}_{6,14}^{a=b=0}, \mathbf{k}_{1,1}=\left\langle e_{2}, e_{4}+e_{1}, e_{5}\right\rangle, \mathbf{k}_{1,2}=\left\langle e_{3}, e_{4}+e_{1}, e_{5}\right\rangle ;$ 


$$
\begin{aligned}
& \mathbf{g}_{2}:=\mathbf{g}_{6,22}^{a=0}, \mathbf{k}_{2}=\left\langle e_{3}, e_{4}+e_{1}, e_{5}\right\rangle, \\
& \mathbf{g}_{3}:=\mathbf{g}_{6,17}^{\delta=1, a=\varepsilon=0}, \mathbf{k}_{3,1}=\left\langle e_{3}, e_{4}, e_{5}+e_{1}\right\rangle, \mathbf{k}_{3,2}=\left\langle e_{2}, e_{4}, e_{5}+e_{1}\right\rangle ; \\
& \mathbf{g}_{4}:=\mathbf{g}_{6,51}^{\varepsilon= \pm 1}, \mathbf{k}_{4}=\left\langle e_{1}+a_{1} e_{2}, e_{3}+e_{2}, e_{4}\right\rangle, a_{1} \in \mathbb{R} \\
& \mathbf{g}_{5}:=\mathbf{g}_{6,54}^{a=b=0}, \mathbf{k}_{5}=\left\langle e_{1}+e_{2}, e_{3}+a_{2} e_{2}, e_{4}\right\rangle, a_{2} \in \mathbb{R} ; \\
& \mathbf{g}_{6}:=\mathbf{g}_{6,63}^{a=0}, \mathbf{k}_{6}=\left\langle e_{1}+e_{2}, e_{3}+a_{2} e_{2}, e_{4}\right\rangle, a_{2} \in \mathbb{R} \text {; } \\
& \mathbf{g}_{7}:=\mathbf{g}_{6,25}^{a=b=0}, \mathbf{k}_{7}=\left\langle e_{1}+e_{5}, e_{2}+\varepsilon e_{5}, e_{4}\right\rangle, \varepsilon=0,1 ; \\
& \mathbf{g}_{8}:=\mathbf{g}_{6,15}^{a=0}, \mathbf{k}_{8}=\left\langle e_{1}+e_{5}, e_{2}+a_{2} e_{5}, e_{4}+a_{3} e_{5}\right\rangle, a_{3} \in \mathbb{R} \backslash\{0\}, a_{2} \in \mathbb{R} ; \\
& \mathbf{g}_{9}:=\mathbf{g}_{6,21}^{a=0,0<|b| \leq 1}, \mathbf{k}_{9}=\left\langle e_{3}, e_{4}+e_{1}, e_{5}+e_{1}\right\rangle ; \\
& \mathbf{g}_{10}:=\mathbf{g}_{6,24}, \mathbf{k}_{10}=\left\langle e_{3}, e_{4}, e_{5}+e_{1}\right\rangle ; \\
& \mathbf{g}_{11}:=\mathbf{g}_{6,30}, \mathbf{k}_{11}=\left\langle e_{3}, e_{4}+a_{2} e_{1}, e_{5}+e_{1}\right\rangle, a_{2} \in \mathbb{R} ; \\
& \mathbf{g}_{12}:=\mathbf{g}_{6,36}^{a=0, b \geq 0}, \mathbf{k}_{12,1}=\left\langle e_{3}, e_{4}, e_{5}+e_{1}\right\rangle, \mathbf{k}_{12,2}=\left\langle e_{3}, e_{4}+e_{1}, e_{5}+a_{3} e_{1}\right\rangle, a_{3} \in \mathbb{R} ; \\
& \mathbf{g}_{13}:=\mathbf{g}_{6,16}, \mathbf{k}_{13}=\left\langle e_{1}+e_{5}, e_{2}+a_{2} e_{5}, e_{4}+a_{3} e_{5}\right\rangle, a_{2}, a_{3} \in \mathbb{R} ; \\
& \mathbf{g}_{14}:=\mathbf{g}_{6,27}^{a=1, b=\delta=0}, \mathbf{k}_{14}=\left\langle e_{1}+e_{5}, e_{2}+a_{2} e_{5}, e_{4}\right\rangle, a_{2} \in \mathbb{R} ; \\
& \mathbf{g}_{15}:=\mathbf{g}_{6,49}^{\varepsilon=0, \pm 1}, \mathbf{k}_{15}=\left\langle e_{1}+a_{1} e_{3}, e_{2}+e_{3}, e_{4}+a_{3} e_{3}\right\rangle, a_{1}, a_{3} \in \mathbb{R} ; \\
& \mathbf{g}_{16}:=\mathbf{g}_{6,52}^{\varepsilon=0, \pm 1}, \mathbf{k}_{16}=\left\langle e_{1}+a_{1} e_{2}, e_{3}+e_{2}, e_{4}\right\rangle, a_{1} \in \mathbb{R} ; \\
& \mathbf{g}_{17}:=\mathbf{g}_{6,57}^{a=0}, \mathbf{k}_{17}=\left\langle e_{1}+e_{2}, e_{3}+a_{2} e_{2}, e_{4}\right\rangle, a_{2} \in \mathbb{R} ; \\
& \mathbf{g}_{18}:=\mathbf{g}_{6,59}^{\delta=1}, \mathbf{k}_{18}=\left\langle e_{1}+e_{2}, e_{3}+a_{2} e_{2}, e_{4}\right\rangle, a_{2} \in \mathbb{R} ; \\
& \mathbf{g}_{19}:=\mathbf{g}_{6,17}^{\delta=\varepsilon=0, a \neq 0}, \mathbf{k}_{19}=\left\langle e_{1}+e_{4}, e_{2}+a_{2} e_{4}, e_{5}+e_{4}\right\rangle, a_{2} \in \mathbb{R} ; \\
& \mathbf{g}_{20}:=\mathbf{g}_{6,17}^{\delta=0, a=\varepsilon=1}, \mathbf{k}_{20}=\left\langle e_{1}+e_{4}, e_{2}+a_{2} e_{4}, e_{5}+a_{3} e_{4}\right\rangle, a_{2}, a_{3} \in \mathbb{R} \text {. }
\end{aligned}
$$

In [11] a single matrix $M$ is established depending on six variables such that the span of the matrices engenders the given Lie algebra in the list $\mathbf{g}_{i}, i=1, \ldots, 20$. To obtain the matrix Lie group $G_{i}$ of the Lie algebra $\mathbf{g}_{i}$ we exponentiate the space of matrices spanned by the matrix $M$. Simplifying the obtained exponential image we get a suitable simple form of a matrix Lie group such that by differentiating and evaluating at the identity its Lie algebra is isomorphic to the Lie algebra $\mathbf{g}_{i}$. In case of the Lie algebras $\mathbf{g}_{j}, j=1,2,8,9,16$, we take in order the exponential image of the matrices:

$$
M_{1}=\left(\begin{array}{cccccc}
0 & -s_{3} & s_{2} & 0 & -s_{6} & 2 s_{1} \\
0 & 0 & 0 & 0 & 0 & s_{2} \\
0 & 0 & 0 & 0 & 0 & s_{3} \\
0 & 0 & 0 & -s_{6} & 0 & s_{4} \\
0 & 0 & 0 & 0 & 0 & 2 s_{5} \\
0 & 0 & 0 & 0 & 0 & 0
\end{array}\right), s_{i} \in \mathbb{R}, i=1, \ldots, 6
$$




$$
\begin{aligned}
& M_{2}=\left(\begin{array}{cccccc}
0 & -s_{3} & s_{2} & 0 & -s_{6} & 2 s_{1} \\
0 & 0 & 0 & 0 & 0 & s_{2} \\
0 & -s_{6} & 0 & 0 & 0 & s_{3} \\
0 & 0 & 0 & -s_{6} & 0 & s_{4} \\
0 & 0 & 0 & 0 & 0 & 2 s_{5} \\
0 & 0 & 0 & 0 & 0 & 0
\end{array}\right), s_{i} \in \mathbb{R}, i=1, \ldots, 6 \\
& M_{8}=\left(\begin{array}{cccccc}
-s_{6} & -s_{3} & -s_{2} & 0 & 0 & 2 s_{1} \\
0 & -s_{6} & 0 & 0 & 0 & s_{2} \\
0 & 0 & 0 & 0 & 0 & -s_{3} \\
0 & -s_{6} & 0 & -s_{6} & 0 & s_{4} \\
0 & 0 & -s_{6} & 0 & 0 & -s_{5} \\
0 & 0 & 0 & 0 & 0 & 0
\end{array}\right), s_{i} \in \mathbb{R}, i=1, \ldots, 6, \\
& M_{9}=\left(\begin{array}{cccccc}
0 & -s_{3} & s_{2} & 0 & 0 & 2 s_{1} \\
0 & 0 & 0 & 0 & 0 & s_{2} \\
0 & -s_{6} & 0 & 0 & 0 & s_{3} \\
0 & 0 & 0 & -s_{6} & 0 & s_{4} \\
0 & 0 & 0 & 0 & -b s_{6} & s_{5} \\
0 & 0 & 0 & 0 & 0 & 0
\end{array}\right), s_{i} \in \mathbb{R}, i=1, \ldots, 6 \\
& M_{16}=\left(\begin{array}{cccccc}
-s_{6} & 0 & 0 & 0 & 0 & s_{3} \\
0 & 0 & 2 s_{5} & -\varepsilon s_{6} & \varepsilon s_{4} & 2 s_{2} \\
0 & 0 & 0 & s_{5} & 0 & -s_{1} \\
0 & 0 & 0 & 0 & s_{5} & s_{4} \\
0 & 0 & 0 & 0 & 0 & s_{6} \\
0 & 0 & 0 & 0 & 0 & 0
\end{array}\right), s_{i} \in \mathbb{R}, \varepsilon=0, \pm 1, i=1, \ldots, 6 \text {. }
\end{aligned}
$$

This procedure yields the following

Theorem 3.1. The simply connected Lie group $G_{i}$ and its subgroup $K_{i}$ of the Lie algebra $\mathbf{g}_{i}$ and its subalgebra $\mathbf{k}_{i}, i=1, \ldots, 20$, is isomorphic to the linear group of matrices the multiplication of which is given by:

For $i=1$ :

$$
\begin{gathered}
g\left(x_{1}, x_{2}, x_{3}, x_{4}, x_{5}, x_{6}\right) g\left(y_{1}, y_{2}, y_{3}, y_{4}, y_{5}, y_{6}\right) \\
=g\left(x_{1}+y_{1}+x_{2} y_{3}-x_{3} y_{2}-x_{6} y_{5}, x_{2}+y_{2}, x_{3}+y_{3}, x_{4}+y_{4} e^{-x_{6}}, x_{5}+y_{5}, x_{6}+y_{6}\right), \\
K_{1,1}=\left\{g\left(u_{1}, u_{3}, 0, u_{1}, u_{2}, 0\right) ; u_{i} \in \mathbb{R}, i=1,2,3\right\}, \\
K_{1,2}=\left\{g\left(u_{1}, 0, u_{3}, u_{1}, u_{2}, 0\right) ; u_{i} \in \mathbb{R}, i=1,2,3\right\},
\end{gathered}
$$

for $i=2$ :

$$
\begin{aligned}
& g\left(x_{1}, x_{2}, x_{3}, x_{4}, x_{5}, x_{6}\right) g\left(y_{1}, y_{2}, y_{3}, y_{4}, y_{5}, y_{6}\right) \\
& =g\left(x_{1}+y_{1}+x_{2} y_{3}-x_{3} y_{2}-x_{6}\left(y_{5}+x_{2} y_{2}\right),\right. \\
& \left.x_{2}+y_{2}, x_{3}+y_{3}-x_{6} y_{2}, x_{4}+y_{4} e^{-x_{6}}, x_{5}+y_{5}, x_{6}+y_{6}\right), \\
& K_{2}=\left\{g\left(u_{1}, 0, u_{3}, u_{1}, u_{2}, 0\right) ; u_{i} \in \mathbb{R}, i=1,2,3\right\}
\end{aligned}
$$


for $i=3$ :

$$
\begin{aligned}
& g\left(x_{1}, x_{2}, x_{3}, x_{4}, x_{5}, x_{6}\right) g\left(y_{1}, y_{2}, y_{3}, y_{4}, y_{5}, y_{6}\right) \\
& =g\left(x_{1}+y_{1}-x_{6} y_{4}+\left(\frac{1}{2} x_{6}^{2}+x_{3}\right) y_{2},\right. \\
& \left.x_{2}+y_{2}, x_{3}+y_{3}, x_{4}+y_{4}-x_{6} y_{2}, x_{5}+y_{5} e^{-x_{6}}, x_{6}+y_{6}\right), \\
& K_{3,1}=\left\{g\left(u_{2}, u_{3}, 0, u_{1}, u_{2}, 0\right) ; u_{i} \in \mathbb{R}, i=1,2,3\right\}, \\
& K_{3,2}=\left\{g\left(u_{2}, 0, u_{3}, u_{1}, u_{2}, 0\right) ; u_{i} \in \mathbb{R}, i=1,2,3\right\},
\end{aligned}
$$

for $i=4$ :

$$
\begin{gathered}
g\left(x_{1}, x_{2}, x_{3}, x_{4}, x_{5}, x_{6}\right) g\left(y_{1}, y_{2}, y_{3}, y_{4}, y_{5}, y_{6}\right) \\
=g\left(x_{1}+y_{1}+x_{5} y_{4}, x_{2}+y_{2}+x_{5} y_{1}+\varepsilon x_{4} y_{6}+\frac{1}{2} x_{5}^{2} y_{4},\right. \\
\left.x_{3}+y_{3} e^{-x_{6}}, x_{4}+y_{4}, x_{5}+y_{5}, x_{6}+y_{6}\right), \varepsilon= \pm 1, \\
K_{4}=\left\{g\left(u_{1}, a_{1} u_{1}+u_{2}, u_{2}, u_{3}, 0,0\right) ; u_{i} \in \mathbb{R}, i=1,2,3\right\}, a_{1} \in \mathbb{R},
\end{gathered}
$$

for $i=5$ :

$$
\begin{gathered}
g\left(x_{1}, x_{2}, x_{3}, x_{4}, x_{5}, x_{6}\right) g\left(y_{1}, y_{2}, y_{3}, y_{4}, y_{5}, y_{6}\right) \\
=g\left(x_{1}+\left(y_{1}+x_{5} y_{3}\right) e^{-x_{6}}, x_{2}+y_{2}+x_{5} y_{4}, x_{3}+y_{3} e^{-x_{6}}, x_{4}+y_{4}, x_{5}+y_{5}, x_{6}+y_{6}\right), \\
K_{5}=\left\{g\left(u_{1}, u_{1}+a_{2} u_{2}, u_{2}, u_{3}, 0,0\right) ; u_{i} \in \mathbb{R}, i=1,2,3\right\}, a_{2} \in \mathbb{R},
\end{gathered}
$$

for $i=6$ :

$$
\begin{aligned}
& g\left(x_{1}, x_{2}, x_{3}, x_{4}, x_{5}, x_{6}\right) g\left(y_{1}, y_{2}, y_{3}, y_{4}, y_{5}, y_{6}\right) \\
& =g\left(x_{1}+\left(y_{1}+y_{3} x_{5}\right) e^{-x_{6}},\right. \\
& \left.\quad x_{2}+y_{2}-\left(x_{5}+x_{6}\right) y_{4}, x_{3}+y_{3} e^{-x_{6}}, x_{4}+y_{4}, x_{5}+y_{5}, x_{6}+y_{6}\right), \\
& K_{6}=\left\{g\left(u_{1}, u_{1}+a_{2} u_{2}, u_{2}, u_{3}, 0,0\right) ; u_{i} \in \mathbb{R}, i=1,2,3\right\}, a_{2} \in \mathbb{R},
\end{aligned}
$$

for $i=7$ :

$$
\begin{gathered}
g\left(x_{1}, x_{2}, x_{3}, x_{4}, x_{5}, x_{6}\right) g\left(y_{1}, y_{2}, y_{3}, y_{4}, y_{5}, y_{6}\right) \\
=g\left(x_{1}+\left(y_{1}+y_{2} x_{3}\right) e^{-x_{6}}, x_{2}+y_{2} e^{-x_{6}}, x_{3}+y_{3}, x_{4}+y_{4}, x_{5}+y_{5}-x_{4} y_{6}, x_{6}+y_{6}\right), \\
K_{7}=\left\{g\left(u_{1}, u_{2}, 0, u_{3}, u_{1}+\varepsilon u_{2}, 0\right) ; u_{i} \in \mathbb{R}, i=1,2,3\right\}, \varepsilon=0,1,
\end{gathered}
$$

for $i=8$ :

$$
\begin{aligned}
& g\left(x_{1}, x_{2}, x_{3}, x_{4}, x_{5}, x_{6}\right) g\left(y_{1}, y_{2}, y_{3}, y_{4}, y_{5}, y_{6}\right) \\
= & g\left(x_{1}+\left(y_{1}+y_{2} x_{3}\right) e^{-x_{6}}-y_{3} x_{2},\right. \\
& \left.x_{2}+y_{2} e^{-x_{6}}, x_{3}+y_{3}, x_{4}+\left(y_{4}-y_{2} x_{6}\right) e^{-x_{6}}, x_{5}+y_{5}-x_{6} y_{3}, x_{6}+y_{6}\right), \\
K_{8}= & \left\{g\left(u_{1}, u_{2}, 0, u_{3}, u_{1}+a_{2} u_{2}+a_{3} u_{3}, 0\right) ; u_{i} \in \mathbb{R}, i=1,2,3\right\}, a_{3} \in \mathbb{R} \backslash\{0\}, a_{2} \in \mathbb{R},
\end{aligned}
$$


for $i=9$ :

$$
\begin{aligned}
& g\left(x_{1}, x_{2}, x_{3}, x_{4}, x_{5}, x_{6}\right) g\left(y_{1}, y_{2}, y_{3}, y_{4}, y_{5}, y_{6}\right) \\
& =g\left(x_{1}+y_{1}+x_{2} y_{3}-\left(x_{3}+x_{2} x_{6}\right) y_{2}, x_{2}+y_{2},\right. \\
& \left.x_{3}+y_{3}-x_{6} y_{2}, x_{4}+y_{4} e^{-x_{6}}, x_{5}+y_{5} e^{-b x_{6}}, x_{6}+y_{6}\right), 0<|b| \leq 1, \\
& K_{9}=\left\{g\left(u_{1}+u_{2}, 0, u_{3}, u_{1}, u_{2}, 0\right) ; u_{i} \in \mathbb{R}, i=1,2,3\right\},
\end{aligned}
$$

for $i=10$ :

$$
\begin{gathered}
g\left(x_{1}, x_{2}, x_{3}, x_{4}, x_{5}, x_{6}\right) g\left(y_{1}, y_{2}, y_{3}, y_{4}, y_{5}, y_{6}\right) \\
=g\left(x_{1}+y_{1}-2 x_{6} y_{4}+\left(x_{6}^{2}-x_{2}\right) y_{3}-\left(\frac{1}{3} x_{6}^{3}-x_{2} x_{6}-x_{3}\right) y_{2}, x_{2}+y_{2},\right. \\
\left.x_{3}+y_{3}-x_{6} y_{2}, x_{4}+y_{4}-x_{6} y_{3}+\frac{1}{2} x_{6}^{2} y_{2}, x_{5}+y_{5} e^{-x_{6}}, x_{6}+y_{6}\right), \\
K_{10}=\left\{g\left(u_{2}, 0, u_{3}, u_{1}, u_{2}, 0\right) ; u_{i} \in \mathbb{R}, i=1,2,3\right\},
\end{gathered}
$$

for $i=11$ :

$$
\begin{gathered}
g\left(x_{1}, x_{2}, x_{3}, x_{4}, x_{5}, x_{6}\right) g\left(y_{1}, y_{2}, y_{3}, y_{4}, y_{5}, y_{6}\right) \\
=g\left(x_{1}+y_{1}+x_{2} y_{3}-\frac{1}{2} x_{2}^{2} y_{6}, x_{2}+y_{2}, x_{3}+y_{3}-x_{2} y_{6},\right. \\
\left.x_{4}+y_{4} e^{-x_{6}}, x_{5}+y_{5} e^{-x_{6}}-x_{4} y_{6}, x_{6}+y_{6}\right), \\
K_{11}=\left\{g\left(a_{2} u_{1}+u_{2}, 0, u_{3}, u_{1}, u_{2}, 0\right) ; u_{i} \in \mathbb{R}, i=1,2,3\right\}, a_{2} \in \mathbb{R},
\end{gathered}
$$

for $i=12$ :

$$
\begin{aligned}
g\left(x_{1}, x_{2}, x_{3}, x_{4}, x_{5}, x_{6}\right) g\left(y_{1}, y_{2}, y_{3}, y_{4}, y_{5}, y_{6}\right) & \\
= & g\left(x_{1}+y_{1}-x_{2} y_{3}+y_{2}\left(x_{3}+x_{2} x_{6}\right), x_{2}+y_{2}, x_{3}+y_{3}-x_{6} y_{2},\right. \\
& x_{4}+y_{4} e^{-b x_{6}} \cos x_{6}+y_{5} e^{-b x_{6}} \sin x_{6}, \\
& \left.x_{5}-y_{4} e^{-b x_{6}} \sin x_{6}+y_{5} e^{-b x_{6}} \cos x_{6}, x_{6}+y_{6}\right), b \geq 0, \\
K_{12,1}= & \left\{g\left(u_{2}, 0, u_{3}, u_{1}, u_{2}, 0\right) ; u_{i} \in \mathbb{R}, i=1,2,3\right\}, \\
K_{12,2}= & \left\{g\left(u_{1}+a_{3} u_{2}, 0, u_{3}, u_{1}, u_{2}, 0\right) ; u_{i} \in \mathbb{R}, i=1,2,3\right\}, a_{3} \in \mathbb{R},
\end{aligned}
$$

for $i=13$ :

$$
\begin{gathered}
g\left(x_{1}, x_{2}, x_{3}, x_{4}, x_{5}, x_{6}\right) g\left(y_{1}, y_{2}, y_{3}, y_{4}, y_{5}, y_{6}\right) \\
=g\left(x_{1}+\left[y_{1}-y_{4} x_{6}+y_{2}\left(\frac{1}{2} x_{6}^{2}+x_{3}\right)\right] e^{-x_{6}}-x_{2} y_{3}, x_{2}+y_{2} e^{-x_{6}},\right. \\
\left.x_{3}+y_{3}, x_{4}+\left(y_{4}-y_{2} x_{6}\right) e^{-x_{6}}, x_{5}+y_{5}-x_{6} y_{3}, x_{6}+y_{6}\right), \\
K_{13}=\left\{g\left(u_{1}, u_{2}, 0, u_{3}, u_{1}+a_{2} u_{2}+a_{3} u_{3}, 0\right) ; u_{i} \in \mathbb{R}, i=1,2,3\right\}, a_{2}, a_{3} \in \mathbb{R},
\end{gathered}
$$

for $i=14$ :

$$
\begin{aligned}
& g\left(x_{1}, x_{2}, x_{3}, x_{4}, x_{5}, x_{6}\right) g\left(y_{1}, y_{2}, y_{3}, y_{4}, y_{5}, y_{6}\right) \\
& =g\left(x_{1}+y_{1} e^{-x_{6}}+x_{2} y_{3}, x_{2}+y_{2} e^{-x_{6}}, x_{3}+y_{3},\right. \\
& \left.\quad x_{4}+y_{4}-x_{6} y_{3}, x_{5}+y_{5}-x_{6} y_{4}+\frac{1}{2} x_{6}^{2} y_{3}, x_{6}+y_{6}\right),
\end{aligned}
$$


$K_{14}=\left\{g\left(u_{1}, u_{2}, 0, u_{3}, u_{1}+a_{2} u_{2}, 0\right) ; u_{i} \in \mathbb{R}, i=1,2,3\right\}, a_{2} \in \mathbb{R}$,

for $i=15$ :

$$
\begin{aligned}
& g\left(x_{1}, x_{2}, x_{3}, x_{4}, x_{5}, x_{6}\right) g\left(y_{1}, y_{2}, y_{3}, y_{4}, y_{5}, y_{6}\right) \\
& =g\left(x_{1}+y_{1} e^{-x_{6}}+x_{4} y_{5}, x_{2}+\left(y_{2}-2 \varepsilon y_{4} x_{6}-y_{1} x_{5}\right) e^{-x_{6}}+\left(x_{1}-x_{4} x_{5}\right) y_{5},\right. \\
& \left.\quad x_{3}+y_{3}-x_{6} y_{5}, x_{4}+y_{4} e^{-x_{6}}, x_{5}+y_{5}, x_{6}+y_{6}\right), \varepsilon=0, \pm 1, \\
& K_{15}=\left\{g\left(u_{1}, u_{2}, a_{1} u_{1}+u_{2}+a_{3} u_{3}, u_{3}, 0,0\right) ; u_{i} \in \mathbb{R}, i=1,2,3\right\}, a_{1}, a_{3} \in \mathbb{R},
\end{aligned}
$$

for $i=16$ :

$$
\begin{aligned}
& g\left(x_{1}, x_{2}, x_{3}, x_{4}, x_{5}, x_{6}\right) g\left(y_{1}, y_{2}, y_{3}, y_{4}, y_{5}, y_{6}\right) \\
& =g\left(x_{1}+y_{1}+x_{5} y_{4}+\frac{1}{2} x_{5}^{2} y_{6},\right. \\
& x_{2}+y_{2}+2 x_{5} y_{1}+\left(x_{5}^{2}-\varepsilon x_{6}\right) y_{4}+\left(\frac{1}{3} x_{5}^{3}+\varepsilon\left(x_{4}-x_{5} x_{6}\right)\right) y_{6}, \\
& \left.x_{3}+y_{3} e^{-x_{6}}, x_{4}+y_{4}+x_{5} y_{6}, x_{5}+y_{5}, x_{6}+y_{6}\right), \varepsilon=0, \pm 1, \\
& K_{16}=\left\{g\left(u_{1}, a_{1} u_{1}+u_{2}, u_{2}, u_{3}, 0,0\right) ; u_{i} \in \mathbb{R}, i=1,2,3\right\}, a_{1} \in \mathbb{R},
\end{aligned}
$$

for $i=17$ :

$$
\begin{gathered}
g\left(x_{1}, x_{2}, x_{3}, x_{4}, x_{5}, x_{6}\right) g\left(y_{1}, y_{2}, y_{3}, y_{4}, y_{5}, y_{6}\right) \\
=g\left(x_{1}+\left(y_{1}+x_{5} y_{3}\right) e^{-x_{6}}, x_{2}+y_{2}+x_{5} y_{4}-\frac{1}{2} x_{5}^{2} y_{6},\right. \\
\left.x_{3}+y_{3} e^{-x_{6}}, x_{4}+y_{4}-x_{5} y_{6}, x_{5}+y_{5}, x_{6}+y_{6}\right), \\
K_{17}=\left\{g\left(u_{1}, u_{1}+a_{2} u_{2}, u_{2}, u_{3}, 0,0\right) ; u_{i} \in \mathbb{R}, i=1,2,3\right\}, a_{2} \in \mathbb{R},
\end{gathered}
$$

for $i=18$ :

$$
\begin{aligned}
& g\left(x_{1}, x_{2}, x_{3}, x_{4}, x_{5}, x_{6}\right) g\left(y_{1}, y_{2}, y_{3}, y_{4}, y_{5}, y_{6}\right) \\
& =g\left(x_{1}+\left(y_{1}+y_{3} x_{5}\right) e^{-x_{6}}, x_{2}+y_{2}-\left(x_{5}+x_{6}\right) y_{4}-\frac{1}{2}\left(x_{5}+x_{6}\right)^{2} y_{5},\right. \\
& \left.x_{3}+y_{3} e^{-x_{6}}, x_{4}+y_{4}+\left(x_{5}+x_{6}\right) y_{5}, x_{5}+y_{5}, x_{6}+y_{6}\right), \\
& K_{18}=\left\{g\left(u_{1}, u_{1}+a_{2} u_{2}, u_{2}, u_{3}, 0,0\right) ; u_{i} \in \mathbb{R}, i=1,2,3\right\}, a_{2} \in \mathbb{R},
\end{aligned}
$$

for $i=19$ :

$$
\begin{aligned}
& g\left(x_{1}, x_{2}, x_{3}, x_{4}, x_{5}, x_{6}\right) g\left(y_{1}, y_{2}, y_{3}, y_{4}, y_{5}, y_{6}\right) \\
& =g\left(x_{1}+y_{1} e^{-a x_{6}}+x_{3} y_{2}, x_{2}+y_{2}, x_{3}+y_{3} e^{-a x_{6}},\right. \\
& \left.\quad x_{4}+y_{4}-x_{6} y_{2}, x_{5}+y_{5} e^{-x_{6}}, x_{6}+y_{6}\right), a \in \mathbb{R} \backslash\{0\},
\end{aligned}
$$

$K_{19}=\left\{g\left(u_{1}, 0, u_{2}, u_{1}+a_{2} u_{2}+u_{3}, u_{3}, 0\right) ; u_{i} \in \mathbb{R}, i=1,2,3\right\}, a_{2} \in \mathbb{R}$,

for $i=20$ :

$$
\begin{gathered}
g\left(x_{1}, x_{2}, x_{3}, x_{4}, x_{5}, x_{6}\right) g\left(y_{1}, y_{2}, y_{3}, y_{4}, y_{5}, y_{6}\right) \\
=g\left(x_{1}+\left(y_{1}-x_{6} y_{5}+y_{2} x_{3}\right) e^{-x_{6}}, x_{2}+y_{2} e^{-x_{6}},\right. \\
\left.x_{3}+y_{3}, x_{4}+y_{4}-x_{3} y_{6}, x_{5}+y_{5} e^{-x_{6}}, x_{6}+y_{6}\right), \\
K_{20}=\left\{g\left(u_{1}, u_{2}, 0, u_{1}+a_{2} u_{2}+a_{3} u_{3}, u_{3}, 0\right) ; u_{i} \in \mathbb{R}, i=1,2,3\right\}, a_{2}, a_{3} \in \mathbb{R} .
\end{gathered}
$$


Among the Lie groups in Theorem 3.1 only the group $G_{1}$ has 2-dimensional commutator subgroup and the groups $G_{i}, i=2, \ldots, 7$, have 3 -dimensional commutator subgroup. We show that among the 6-dimensional solvable indecomposable Lie groups with 5-dimensional nilradical precisely these Lie groups are the multiplication groups of three-dimensional connected simply connected topological loops.

Proposition 3.2. There does not exist 3-dimensional connected topological proper loop L such that the Lie algebra $\mathbf{g}$ of the multiplication group of $L$ is one of the Lie algebras $\mathbf{g}_{i}, i=8, \ldots, 20$.

Proof. If $L$ exists, then there exists its universal covering loop $\tilde{L}$ which is homeomorphic to $\mathbb{R}^{3}$. The pairs $\left(G_{i}, K_{i}\right)$ in Theorem 3.1 can occur as the group $M u l t(\tilde{L})$ and the subgroup $\operatorname{Inn}(\tilde{L})$. We show that none of the groups $G_{i}, i=8, \ldots, 20$, satisfies the condition that there exist continuous left transversals $A$ and $B$ to $K_{i}$ in $G_{i}$ such that for all $a \in A$ and $b \in B$ one has $a^{-1} b^{-1} a b \in K_{i}$. By Proposition 2.1 the groups $G_{i}, i=8, \ldots, 20$, are not the multiplication group of a loop $\tilde{L}$. Hence no proper loop $\tilde{L}$ exists which yields that also no proper loop $L$ exists. This proves the assertion.

Two arbitrary left transversals to the group $K_{i}$ in $G_{i}$ are:

For $i=9,10,11,12$,

$$
\begin{aligned}
& A=\left\{g\left(u, v, h_{1}(u, v, w), h_{2}(u, v, w), h_{3}(u, v, w), w\right) ; u, v, w \in \mathbb{R}\right\}, \\
& B=\left\{g\left(k, l, f_{1}(k, l, m), f_{2}(k, l, m), f_{3}(k, l, m), m\right) ; k, l, m \in \mathbb{R}\right\},
\end{aligned}
$$

for $i=8,13,14,15$,

$$
\begin{aligned}
& A=\left\{g\left(h_{1}(u, v, w), h_{2}(u, v, w), u, h_{3}(u, v, w), v, w\right) ; u, v, w \in \mathbb{R}\right\}, \\
& B=\left\{g\left(f_{1}(k, l, m), f_{2}(k, l, m), k, f_{3}(k, l, m), l, m\right) ; k, l, m \in \mathbb{R}\right\},
\end{aligned}
$$

for $i=16,17,18$,

$$
\begin{aligned}
& A=\left\{g\left(h_{1}(u, v, w), u, h_{2}(u, v, w), h_{3}(u, v, w), v, w\right) ; u, v, w \in \mathbb{R}\right\}, \\
& B=\left\{g\left(f_{1}(k, l, m), k, f_{2}(k, l, m), f_{3}(k, l, m), l, m\right) ; k, l, m \in \mathbb{R}\right\},
\end{aligned}
$$

for $i=19$

$$
\begin{aligned}
& A=\left\{g\left(h_{1}(u, v, w), u, h_{2}(u, v, w), v, h_{3}(u, v, w), w\right) ; u, v, w \in \mathbb{R}\right\}, \\
& B=\left\{g\left(f_{1}(k, l, m), k, f_{2}(k, l, m), l, f_{3}(k, l, m), m\right) ; k, l, m \in \mathbb{R}\right\},
\end{aligned}
$$

for $i=20$

$$
\begin{aligned}
& A=\left\{g\left(h_{1}(u, v, w), h_{2}(u, v, w), u, v, h_{3}(u, v, w), w\right) ; u, v, w \in \mathbb{R}\right\}, \\
& B=\left\{g\left(f_{1}(k, l, m), f_{2}(k, l, m), k, l, f_{3}(k, l, m), m\right) ; k, l, m \in \mathbb{R}\right\},
\end{aligned}
$$

where $h_{i}(u, v, w): \mathbb{R}^{3} \rightarrow \mathbb{R}$ and $f_{i}(k, l, m): \mathbb{R}^{3} \rightarrow \mathbb{R}, i=1,2,3$, are continuous functions with $f_{i}(0,0,0)=h_{i}(0,0,0)=0$. Taking in $G_{i}, i=9,11,12$, the elements

$$
a=g\left(0, v, h_{1}(0, v, 0), h_{2}(0, v, 0), h_{3}(0, v, 0), 0\right) \in A,
$$




$$
b=g\left(0,0, f_{1}(0,0, m), f_{2}(0,0, m), f_{3}(0,0, m), m\right) \in B
$$

and in $G_{17}$ the elements

$$
\begin{aligned}
a & =g\left(h_{1}(0, v, 0), 0, h_{2}(0, v, 0), h_{3}(0, v, 0), v, 0\right) \in A, \\
b & =g\left(f_{1}(0,0, m), 0, f_{2}(0,0, m), f_{3}(0,0, m), 0, m\right) \in B
\end{aligned}
$$

one has $a^{-1} b^{-1} a b \in K_{i}$ if and only if

for $i=9$

$$
m v^{2}-2 v f_{1}(0,0, m)=h_{2}(0, v, 0)\left(1-e^{m}\right)+h_{3}(0, v, 0)\left(1-e^{b m}\right),
$$

for $i=11$

$$
\frac{1}{2} m v^{2}+v f_{1}(0,0, m)=\left(e^{m}-1\right)\left(h_{3}(0, v, 0)+a_{2} h_{2}(0, v, 0)\right)-e^{m} m h_{2}(0, v, 0),
$$

for $i=12$ and for $K_{12,1}$

$$
2 v f_{1}(0,0, m)-m v^{2}=\left(1-e^{b m} \cos m\right) h_{3}(0, v, 0)-e^{b m} \sin m h_{2}(0, v, 0),
$$

for $i=12$ and for $K_{12,2}$

$$
\begin{aligned}
2 v f_{1}(0,0, m)-m v^{2}= & \left(1-e^{b m} \cos m\right)\left(h_{2}(0, v, 0)+a_{3} h_{3}(0, v, 0)\right) \\
& +e^{b m} \sin m\left(h_{3}(0, v, 0)-a_{3} h_{2}(0, v, 0)\right),
\end{aligned}
$$

for $i=17$

$$
\begin{aligned}
-\frac{1}{2} m v^{2}-v f_{3}(0,0, m)= & \left(1-e^{m}\right)\left[h_{1}(0, v, 0)+\left(a_{2}-v\right) h_{2}(0, v, 0)\right] \\
& -e^{m} v f_{2}(0,0, m)
\end{aligned}
$$

is satisfied for all $m, v \in \mathbb{R}$. On the left hand side of equations (3.1), (3.2), (3.3), $(3.4),(3.5)$ is the term $m v^{2}$ hence there does not exist any function $f_{i}(0,0, m)$ and $h_{i}(0, v, 0), i=1,2,3$, satisfying these equations. Taking in $G_{10}$ the elements

$$
\begin{aligned}
& a=g\left(0, v, h_{1}(0, v, w), h_{2}(0, v, w), h_{3}(0, v, w), w\right) \in A \\
& b=g\left(0,0, f_{1}(0,0, m), f_{2}(0,0, m), f_{3}(0,0, m), m\right) \in B,
\end{aligned}
$$

respectively in $G_{18}$ the elements

$$
\begin{aligned}
& a=g\left(h_{1}(0, v, w), 0, h_{2}(0, v, w), h_{3}(0, v, w), v, w\right) \in A, \\
& b=g\left(f_{1}(0,0, m), 0, f_{2}(0,0, m), f_{3}(0,0, m), 0, m\right) \in B,
\end{aligned}
$$

respectively in $G_{16}$ the elements

$$
\begin{aligned}
& a=g\left(h_{1}(0, v, 0), 0, h_{2}(0, v, 0), h_{3}(0, v, 0), v, 0\right) \in A, \\
& b=g\left(f_{1}(0, l, m), 0, f_{2}(0, l, m), f_{3}(0, l, m), l, m\right) \in B
\end{aligned}
$$


we obtain that $a^{-1} b^{-1} a b \in K_{i}$ if and only if in case $i=10$ the equation

$$
\begin{aligned}
e^{w}\left(1-e^{m}\right) h_{3} & (0, v, w)+e^{m}\left(e^{w}-1\right) f_{3}(0,0, m) \\
= & \left(w^{2}+2 v+2 m w\right) f_{1}(0,0, m)+2 w f_{2}(0,0, m) \\
& -\left(m^{2}+2 w m\right) h_{1}(0, v, w)-2 m h_{2}(0, v, w) \\
& -m^{2} w v-w^{2} m v-m v^{2}-\frac{1}{3} v m^{3}
\end{aligned}
$$

respectively in case $i=18$ the equation

$$
\begin{aligned}
& e^{m}\left(e^{w}-1\right)\left(f_{1}(0,0, m)+a_{2} f_{2}(0,0, m)\right) \\
& \quad+e^{w}\left(1-e^{m}\right)\left[h_{1}(0, v, w)+\left(a_{2}-v\right) h_{2}(0, v, w)\right] \\
& =e^{m+w} v f_{2}(0,0, m)+(w+v) f_{3}(0,0, m) \\
& \quad-m h_{3}(0, v, w)+v^{2} m+\frac{1}{2} m^{2} v+w v m,
\end{aligned}
$$

respectively in case $i=16$ the equation

$$
\begin{aligned}
- & \frac{1}{3} v^{3} m-v^{2} l m-l^{2} v m-\frac{1}{2} a_{1} v^{2} m-\varepsilon m^{2} v-a_{1} v l m \\
= & \left(1-e^{m}\right) h_{2}(0, v, 0)-2 l h_{1}(0, v, 0)+\left(l^{2}+2 v l+a_{1} l+2 \varepsilon m\right) h_{3}(0, v, 0) \\
& +2 v f_{1}(0, l, m)-\left(v^{2}+2 v l+a_{1} v\right) f_{3}(0, l, m)
\end{aligned}
$$

holds for all $m, l, v, w \in \mathbb{R}$. Substituting into (3.6)

$$
f_{2}(0,0, m)=f_{2}^{\prime}(0,0, m)-m f_{1}(0,0, m), h_{2}(0, v, w)=h_{2}^{\prime}(0, v, w)-w h_{1}(0, v, w),
$$

respectively into $(3.7)$

$f_{1}(0,0, m)=f_{1}^{\prime}(0,0, m)-a_{2} f_{2}(0,0, m), h_{1}(0, v, w)=h_{1}^{\prime}(0, v, w)+\left(v-a_{2}\right) h_{2}(0, v, w)$, respectively into (3.8)

$$
\begin{aligned}
& h_{1}(0, v, 0)=h_{1}^{\prime}(0, v, 0)+\left(v+\frac{1}{2} a_{1}\right) h_{3}(0, v, 0), \\
& f_{1}(0, l, m)=f_{1}^{\prime}(0, l, m)+\left(l+\frac{1}{2} a_{1}\right) f_{3}(0, l, m),
\end{aligned}
$$

we get in case $i=10$

$$
\begin{aligned}
& e^{w}\left(1-e^{m}\right) h_{3}(0, v, w)+e^{m}\left(e^{w}-1\right) f_{3}(0,0, m) \\
& =\left(w^{2}+2 v\right) f_{1}(0,0, m)-m^{2} h_{1}(0, v, w)+2 w f_{2}^{\prime}(0,0, m) \\
& \quad-2 m h_{2}^{\prime}(0, v, w)-m^{2} w v-w^{2} m v-m v^{2}-\frac{1}{3} v m^{3}
\end{aligned}
$$

respectively in case $i=18$

$$
\begin{aligned}
& e^{m}\left(e^{w}-1\right) f_{1}^{\prime}(0,0, m)-e^{m+w} v f_{2}(0,0, m)+e^{w}\left(1-e^{m}\right) h_{1}^{\prime}(0, v, w) \\
& =(w+v) f_{3}(0,0, m)-m h_{3}(0, v, w)+v^{2} m+\frac{1}{2} m^{2} v+w v m,
\end{aligned}
$$


respectively in case $i=16$

$$
\begin{aligned}
& \left(1-e^{m}\right) h_{2}(0, v, 0)+\left(l^{2}+2 \varepsilon m\right) h_{3}(0, v, 0) \\
& \quad-v^{2} f_{3}(0, l, m)-2 l h_{1}^{\prime}(0, v, 0)+2 v f_{1}^{\prime}(0, l, m) \\
= & -\frac{1}{3} v^{3} m-v^{2} l m-l^{2} v m-\frac{1}{2} a_{1} v^{2} m-\varepsilon m^{2} v-a_{1} v l m .
\end{aligned}
$$

Since on the right hand side of (3.9), respectively (3.10), respectively (3.11) there is the term $-\frac{1}{3} v m^{3}$, respectively $\frac{1}{2} m^{2} v$, respectively $-\frac{1}{3} v^{3} m$ there does not exist any function $f_{i}(0,0, m)$ and $h_{i}(0, v, w), i=1,2,3$, respectively $f_{i}(0, l, m), i=1,3$, and $h_{j}(0, v, 0), j=1,2,3$, satisfying equation (3.9), respectively (3.10), respectively (3.11).

Taking in $G_{i}, i=8,13,14$, the elements

$$
\begin{aligned}
& a=g\left(h_{1}(0,0, w), h_{2}(0,0, w), 0, h_{3}(0,0, w), 0, w\right) \in A, \\
& b=g\left(f_{1}(k, 0, m), f_{2}(k, 0, m), k, f_{3}(k, 0, m), 0, m\right) \in B,
\end{aligned}
$$

respectively in $G_{19}$ the elements

$$
\begin{aligned}
& a=g\left(h_{1}(0,0, w), 0, h_{2}(0,0, w), 0, h_{3}(0,0, w), w\right) \in A, \\
& b=g\left(f_{1}(k, 0, m), k, f_{2}(k, 0, m), 0, f_{3}(k, 0, m), m\right) \in B,
\end{aligned}
$$

respectively in $G_{20}$ the elements

$$
\begin{aligned}
& a=g\left(h_{1}(0,0, w), h_{2}(0,0, w), 0,0, h_{3}(0,0, w), w\right) \in A, \\
& b=g\left(f_{1}(k, 0, m), f_{2}(k, 0, m), k, 0, f_{3}(k, 0, m), m\right) \in B
\end{aligned}
$$

we have $a^{-1} b^{-1} a b \in K_{i}$ precisely if for $i=8$ the equation

$$
\begin{aligned}
w k= & e^{w}\left(1-e^{m}\right)\left[\left(a_{2}+a_{3} w\right) h_{2}(0,0, w)+a_{3} h_{3}(0,0, w)+h_{1}(0,0, w)\right] \\
& +e^{m}\left(e^{w}-1\right)\left[\left(a_{3} m+a_{2}-k\right) f_{2}(k, 0, m)+a_{3} f_{3}(k, 0, m)+f_{1}(k, 0, m)\right] \\
& +e^{m+w}\left[a_{3} w f_{2}(k, 0, m)+\left(2 k-a_{3} m\right) h_{2}(0,0, w)\right],
\end{aligned}
$$

for $i=13$ the equation

$$
\begin{aligned}
& w k=e^{w}\left(1-e^{m}\right)\left[\left(\frac{1}{2} w^{2}+a_{2}+a_{3} w\right) h_{2}(0,0, w)+\left(a_{3}+w\right) h_{3}(0,0, w)+h_{1}(0,0, w)\right] \\
& +e^{m}\left(e^{w}-1\right)\left[\left(\frac{1}{2} m^{2}-k+a_{3} m+a_{2}\right) f_{2}(k, 0, m)\right. \\
& \left.+\left(m+a_{3}\right) f_{3}(k, 0, m)+f_{1}(k, 0, m)\right] \\
& +e^{m+w}\left[\left(\left(m+a_{3}\right) w+\frac{1}{2} w^{2}\right) f_{2}(k, 0, m)+\left(2 k-\frac{1}{2} m^{2}-\left(w+a_{3}\right) m\right) h_{2}(0,0, w)\right] \\
& +e^{m+w}\left(w f_{3}(k, 0, m)-m h_{3}(0,0, w)\right),
\end{aligned}
$$

for $i=14$ the equation

$$
\begin{aligned}
& \frac{1}{2} w^{2} k+m w k+w f_{3}(k, 0, m)-m h_{3}(0,0, w) \\
& =e^{w}\left(1-e^{m}\right)\left(h_{1}(0,0, w)+a_{2} h_{2}(0,0, w)\right) \\
& \quad+e^{m}\left(e^{w}-1\right)\left(f_{1}(k, 0, m)+a_{2} f_{2}(k, 0, m)\right)-e^{m+w} k h_{2}(0,0, w),
\end{aligned}
$$


for $i=19$ the equation

$$
\begin{aligned}
w k= & e^{w}\left(1-e^{m}\right) h_{3}(0,0, w)-e^{m}\left(1-e^{w}\right) f_{3}(k, 0, m)-e^{a(m+w)} k h_{2}(0,0, w) \\
& +e^{a w}\left(1-e^{a m}\right)\left(h_{1}(0,0, w)+a_{2} h_{2}(0,0, w)\right) \\
& -e^{a m}\left(1-e^{a w}\right)\left(f_{1}(k, 0, m)+a_{2} f_{2}(k, 0, m)\right),
\end{aligned}
$$

for $i=20$ the equation

$$
\begin{aligned}
-w k= & e^{w}\left(1-e^{m}\right)\left(h_{1}(0,0, w)+a_{2} h_{2}(0,0, w)+\left(w+a_{3}\right) h_{3}(0,0, w)\right) \\
& +e^{m}\left(1-e^{w}\right)\left(\left(k-a_{2}\right) f_{2}(k, 0, m)-f_{1}(k, 0, m)-\left(m+a_{3}\right) f_{3}(k, 0, m)\right) \\
& +e^{m+w}\left(k h_{2}(0,0, w)-m h_{3}(0,0, w)+w f_{3}(k, 0, m)\right)
\end{aligned}
$$

is satisfied for all $k, m, w \in \mathbb{R}, a_{2}, a_{3} \in \mathbb{R}$. Putting into (3.12)

$$
\begin{aligned}
& h_{1}(0,0, w)=h_{1}^{\prime}(0,0, w)-\left(a_{3} w+a_{2}\right) h_{2}(0,0, w)-a_{3} h_{3}(0,0, w), \\
& f_{1}(k, 0, m)=f_{1}^{\prime}(k, 0, m)+\left(k-a_{3} m-a_{2}\right) f_{2}(k, 0, m)-a_{3} f_{3}(k, 0, m),
\end{aligned}
$$

respectively into $(3.13)$

$$
\begin{aligned}
& h_{1}(0,0, w)=h_{1}^{\prime}(0,0, w)-\left(\frac{1}{2} w^{2}+a_{3} w+a_{2}\right) h_{2}(0,0, w)-\left(a_{3}+w\right) h_{3}(0,0, w), \\
& f_{1}(k, 0, m)=f_{1}^{\prime}(k, 0, m)+\left(k-\frac{1}{2} m^{2}-a_{3} m-a_{2}\right) f_{2}(k, 0, m)-\left(m+a_{3}\right) f_{3}(k, 0, m), \\
& f_{3}(k, 0, m)=f_{3}^{\prime}(k, 0, m)-\left(m+a_{3}\right) f_{2}(k, 0, m), \\
& h_{3}(0,0, w)=h_{3}^{\prime}(0,0, w)-\left(w+a_{3}\right) h_{2}(0,0, w),
\end{aligned}
$$

respectively into (3.14)

$$
\begin{aligned}
& h_{1}(0,0, w)=h_{1}^{\prime}(0,0, w)-a_{2} h_{2}(0,0, w), \\
& f_{3}(k, 0, m)=f_{3}^{\prime}(k, 0, m)-m k \\
& f_{1}(k, 0, m)=f_{1}^{\prime}(k, 0, m)-a_{2} f_{2}(k, 0, m),
\end{aligned}
$$

respectively into $(3.15)$

$$
\begin{aligned}
& h_{1}(0,0, w)=h_{1}^{\prime}(0,0, w)-a_{2} h_{2}(0,0, w), \\
& f_{1}(k, 0, m)=f_{1}^{\prime}(k, 0, m)-a_{2} f_{2}(k, 0, m),
\end{aligned}
$$

respectively into $(3.16)$

$$
\begin{aligned}
& h_{1}(0,0, w)=h_{1}^{\prime}(0,0, w)-a_{2} h_{2}(0,0, w)-\left(w+a_{3}\right) h_{3}(0,0, w), \\
& f_{1}(k, 0, m)=f_{1}^{\prime}(k, 0, m)+\left(k-a_{2}\right) f_{2}(k, 0, m)-\left(m+a_{3}\right) f_{3}(k, 0, m)
\end{aligned}
$$

in order equations $(3.12),(3.13),(3.14),(3.15),(3.16)$ reduce in case $i=8$ to

$$
\begin{aligned}
w k= & e^{w}\left(1-e^{m}\right) h_{1}^{\prime}(0,0, w)+e^{m}\left(e^{w}-1\right) f_{1}^{\prime}(k, 0, m) \\
& +e^{m+w}\left[a_{3} w f_{2}(k, 0, m)+\left(2 k-a_{3} m\right) h_{2}(0,0, w)\right],
\end{aligned}
$$


in case $i=13$ to

$$
\begin{gathered}
w k=e^{w}\left(1-e^{m}\right) h_{1}^{\prime}(0,0, w)+e^{m}\left(e^{w}-1\right) f_{1}^{\prime}(k, 0, m) \\
+e^{m+w}\left[\frac{1}{2} w^{2} f_{2}(k, 0, m)+\left(2 k-\frac{1}{2} m^{2}\right) h_{2}(0,0, w)\right. \\
\left.+w f_{3}^{\prime}(k, 0, m)-m h_{3}^{\prime}(0,0, w)\right],
\end{gathered}
$$

in case $i=14$ to

$$
\begin{aligned}
& \frac{1}{2} w^{2} k+w f_{3}^{\prime}(k, 0, m)-m h_{3}(0,0, w) \\
& =e^{w}\left(1-e^{m}\right) h_{1}^{\prime}(0,0, w)+e^{m}\left(e^{w}-1\right) f_{1}^{\prime}(k, 0, m)-e^{m+w} k h_{2}(0,0, w),
\end{aligned}
$$

in case $i=19$ to

$$
\begin{aligned}
w k= & e^{w}\left(1-e^{m}\right) h_{3}(0,0, w)-e^{m}\left(1-e^{w}\right) f_{3}(k, 0, m)-e^{a(m+w)} k h_{2}(0,0, w) \\
& +e^{a w}\left(1-e^{a m}\right) h_{1}^{\prime}(0,0, w)-e^{a m}\left(1-e^{a w}\right) f_{1}^{\prime}(k, 0, m),
\end{aligned}
$$

and in case $i=20$ to

$$
\begin{aligned}
-w k= & e^{w}\left(1-e^{m}\right) h_{1}^{\prime}(0,0, w)+e^{m}\left(e^{w}-1\right) f_{1}^{\prime}(k, 0, m) \\
& +e^{m+w}\left(k h_{2}(0,0, w)-m h_{3}(0,0, w)+w f_{3}(k, 0, m)\right) .
\end{aligned}
$$

Since on the left hand side of (3.17), (3.18), (3.20), (3.21), respectively of (3.19) is the term $w k$, respectively $\frac{1}{2} w^{2} k$ there does not exist any function $f_{i}(k, 0, m)$, $h_{i}(0,0, w), i=1,2,3$, satisfying equation (3.17), (3.18), (3.20), (3.21), respectively (3.19).

Taking in $G_{15}$ the elements

$$
\begin{aligned}
a & =g\left(h_{1}(0,0, w), h_{2}(0,0, w), 0, h_{3}(0,0, w), 0, w\right) \in A, \\
b & =g\left(f_{1}(0, l, m), f_{2}(0, l, m), 0, f_{3}(0, l, m), l, m\right) \in B
\end{aligned}
$$

the product $a^{-1} b^{-1} a b$ lies in $K_{15}$ if and only if the equation

$$
\begin{aligned}
w l= & e^{w}\left(1-e^{m}\right)\left[h_{2}(0,0, w)+\left(a_{3}+2 w \varepsilon\right) h_{3}(0,0, w)+a_{1} h_{1}(0,0, w)\right] \\
& +e^{m}\left(e^{w}-1\right)\left[f_{2}(0, l, m)+\left(l+a_{1}\right) f_{1}(0, l, m)+\left(a_{3}+2 m \varepsilon\right) f_{3}(0, l, m)\right] \\
& +e^{m+w}\left[2 w \varepsilon f_{3}(0, l, m)-2 l h_{1}(0,0, w)-\left(l^{2}+2 m \varepsilon+a_{1} l\right) h_{3}(0,0, w)\right]
\end{aligned}
$$

is satisfied for all $m, l, w \in \mathbb{R}$. Substituting into (3.22)

$$
\begin{aligned}
& h_{1}(0,0, w)=h_{1}^{\prime}(0,0, w)-\frac{1}{2} a_{1} h_{3}(0,0, w), \\
& h_{2}(0,0, w)=h_{2}^{\prime}(0,0, w)-a_{1} h_{1}(0,0, w)-\left(a_{3}+2 w \varepsilon\right) h_{3}(0,0, w), \\
& f_{2}(0, l, m)=f_{2}^{\prime}(0, l, m)-\left(l+a_{1}\right) f_{1}(0, l, m)-\left(a_{3}+2 m \varepsilon\right) f_{3}(0, l, m),
\end{aligned}
$$

we obtain

$$
w l=e^{w}\left(1-e^{m}\right) h_{2}^{\prime}(0,0, w)+e^{m}\left(e^{w}-1\right) f_{2}^{\prime}(0, l, m)
$$




$$
+e^{m+w}\left[2 w \varepsilon f_{3}(0, l, m)-2 l h_{1}^{\prime}(0,0, w)-\left(l^{2}+2 m \varepsilon\right) h_{3}(0,0, w)\right] .
$$

On the left hand side of equation (3.23) is the term $w l$ hence there does not exist any function $f_{i}(0, l, m), i=2,3$, and $h_{j}(0,0, w), j=1,2,3$ such that equation (3.23) holds.

Theorem 3.3. Let $L$ be a connected simply connected topological proper loop of dimension 3 such that its multiplication group is a 6-dimensional solvable indecomposable Lie group having 5-dimensional nilradical. Then the pairs of Lie groups $\left(G_{i}, K_{i}\right), i=1, \ldots, 7$, are the multiplication groups $M u l t(L)$ and the inner mapping groups $\operatorname{Inn}(L)$ of $L$.

Proof. The sets

$$
\begin{aligned}
& A=\left\{g\left(k, 1-e^{m}, l, m e^{-m}, 2 l, m\right) ; k, l, m \in \mathbb{R}\right\}, \\
& B=\left\{g\left(u, w, v, 2 v e^{-w}, 1-e^{w}, w\right) ; u, v, w \in \mathbb{R}\right\}
\end{aligned}
$$

respectively

$$
\begin{aligned}
& C=\left\{g\left(k, l, 1-e^{m}, m e^{-m},-2 l, m\right) ; k, l, m \in \mathbb{R}\right\}, \\
& D=\left\{g\left(u, v, w,-2 v e^{-w}, 1-e^{w}, w\right) ; u, v, w \in \mathbb{R}\right\}
\end{aligned}
$$

are $K_{1,1^{-}}$, respectively $K_{1,2^{-}}$connected left transversals in $G_{1}$. The sets

$$
\begin{aligned}
& A=\left\{g\left(k, l, l, m e^{-m}, l^{2}-1+e^{m}, m\right) ; k, l, m \in \mathbb{R}\right\}, \\
& B=\left\{g\left(u, v, v,-w e^{-w}, v^{2}+1-e^{w}, w\right) ; u, v, w \in \mathbb{R}\right\}
\end{aligned}
$$

are $K_{2}$-connected left transversals in $G_{2}$. The sets

$$
\begin{aligned}
& A=\left\{g\left(k, \frac{1}{2} m^{2}-l, l, e^{m}-1-m\left(\frac{1}{2} m^{2}-l\right), m e^{-m}, m\right) ; k, l, m \in \mathbb{R}\right\}, \\
& B=\left\{g\left(u, \frac{1}{2} w^{2}-v, v, 1-e^{w}-w\left(\frac{1}{2} w^{2}-v\right),-w e^{-w}, w\right) ; u, v, w \in \mathbb{R}\right\},
\end{aligned}
$$

respectively

$$
\begin{aligned}
& C=\left\{g\left(k, l, \frac{1}{2} m^{2}+e^{m}-1,-l m+m, l e^{-m}, m\right) ; k, l, m \in \mathbb{R}\right\}, \\
& D=\left\{g\left(u, v, \frac{1}{2} w^{2}-e^{w}+1,-v w+w,-v e^{-w}, w\right) ; u, v, w \in \mathbb{R}\right\}
\end{aligned}
$$

are $K_{3,1^{-}}$, respectively $K_{3,2^{-}}$connected left transversals in $G_{3}$. The sets

$$
\begin{aligned}
& A=\left\{g\left(\left(l+a_{1}\right)\left(1-e^{m}\right)+l, k,-e^{-m}\left(\frac{1}{2} l^{2}+\varepsilon m\right), 1-e^{m}, l, m\right) ; k, l, m \in \mathbb{R}\right\}, \\
& B=\left\{g\left(\left(v+a_{1}\right)\left(e^{w}-1\right)+v, u, e^{-w}\left(\frac{1}{2} v^{2}+\varepsilon w\right), e^{w}-1, v, w\right) ; u, v, w \in \mathbb{R}\right\}
\end{aligned}
$$

are $K_{4}$-connected left transversals in $G_{4}$. The sets

$$
\begin{aligned}
& A=\left\{g\left(l e^{-k}\left(a_{2}-l+1\right), m,-l e^{-k}, 1-l e^{k}-e^{k}, l, k\right) ; k, l, m \in \mathbb{R}\right\}, \\
& B=\left\{g\left(v e^{-u}\left(v-1-a_{2}\right), w, v e^{-u}, v e^{u}+e^{u}-1, v, u\right) ; u, v, w \in \mathbb{R}\right\}
\end{aligned}
$$


are $K_{5}$-connected left transversals in $G_{5}$. The sets

$$
\begin{aligned}
& A=\left\{g\left(\left(l-a_{2}\right) l+(l+m) e^{-m}, k, l, e^{m}-1, l, m\right) ; k, l, m \in \mathbb{R}\right\}, \\
& B=\left\{g\left(\left(v-a_{2}\right) v-(v+w) e^{-w}, u, v, 1-e^{w}, v, w\right) ; u, v, w \in \mathbb{R}\right\}
\end{aligned}
$$

are $K_{6}$-connected left transversals in $G_{6}$. The sets

$$
\begin{aligned}
& A=\left\{g\left((\varepsilon-k) m e^{-m},-m e^{-m}, k,-k e^{m}, l, m\right), k, l, m \in \mathbb{R}\right\}, \\
& B=\left\{g\left((u-\varepsilon) w e^{-w}, w e^{-w}, u, u e^{w}, v, w\right), u, v, w \in \mathbb{R}\right\}
\end{aligned}
$$

are $K_{7}$-connected left transversals in $G_{7}$. For all $i=1, \ldots, 7$, the sets $A, B$, respectively $C, D$ generate the group $G_{i}$. According to Proposition 2.1 the pairs $\left(G_{i}, K_{i}\right), i=1, \ldots, 7$, are multiplication groups and inner mapping groups of $L$ which proves the assertion.

Corollary 3.4. Each 3-dimensional connected topological proper loop L having a solvable indecomposable Lie group of dimension 6 as the group Mult $(L)$ of $L$ has 1-dimensional centre and 2- or 3-dimensional commutator subgroup.

Proof. If $L$ has a 6-dimensional indecomposable nilpotent Lie group as its multiplication group, then the assertion follows from case b) of Theorem in [6]. If it has a 6-dimensional indecomposable solvable Lie group with 4-dimensional nilradical, then the assertion is proved in Theorem 16 in [4]. If it has a 6-dimensional indecomposable solvable Lie group with 5-dimensional nilradical, then Theorems 3.6 and 3.7 in [5] and Theorem 3.3 give the assertion.

\section{References}

[1] A. A. Albert: Quasigroups I, Trans. Amer. Math. Soc. 54 (1943), pp. 507-519.

[2] R. H. Bruck: Contributions to the Theory of Loops, Trans. Amer. Math. Soc. 60 (1946), pp. $245-354$.

[3] Á. Figula: Three-dimensional topological loops with solvable multiplication groups, Comm. Algebra 42 (2014), pp. 444-468.

[4] Á. Figula, A. Al-Aвауеchi: Topological loops having solvable indecomposable Lie groups as their multiplication groups, submitted to Transform. Groups (2018).

[5] Á. Figula, A. Al-Abayechi: Topological loops with solvable multiplication groups of dimension at most six are centrally nilpotent, Int. J. Group Theory (2019), pp. 14, DOI: 10.22108/ijgt.2019.114770.1522.

[6] Á. Figula, M. Lattuca: Three-dimensional topological loops with nilpotent multiplication groups, J. Lie Theory 25 (2015), pp. 787-805.

[7] G. M. Mubarakzyanov: Classification of Solvable Lie Algebras in dimension six with one non-nilpotent basis element, Izv. Vyssh. Uchebn. Zaved. Mat. 4 (1963), pp. 104-116.

[8] P. T. Nagy, K. Strambach: Loops in Group Theory and Lie Theory (De Gruyter Expositions in Mathematics, 35), Berlin: Walter de Gruyter GmbH \& Co. KG, 2002.

[9] M. NiemenmaA, T. Kepka: On Multiplication Groups of Loops, J. Algebra 135 (1990), pp. $112-122$. 
[10] A. Shabanskaya, G. Thompson: Six-dimensional Lie algebras with a five-dimensional nilradical, J. Lie Theory 23 (2013), pp. 313-355.

[11] G. Thompson, C. Hettiarachchi, N. Jones, A. Shabanskaya: Representations of Sixdimensional Mubarakazyanov Lie algebras, J. Gen. Lie Theory Appl. 8.1 (2014), Art. ID 1000211, $10 \mathrm{pp}$. 